\title{
A Mass Spectrometric Study of Kratom Compounds by Direct Infusion Electrospray Ionization Triple Quadrupole Mass Spectrometry
}

\author{
Hanzhuo Fu \\ Ultimate Analysis Laboratory, Boca Raton, USA \\ Email: hanzhuo.fu@gmail.com \\ Received 17 April 2016; accepted 20 June 2016; published 23 June 2016 \\ Copyright (C) 2016 by author and Scientific Research Publishing Inc. \\ This work is licensed under the Creative Commons Attribution International License (CC BY). \\ http://creativecommons.org/licenses/by/4.0/

(c) (i) Open Access

\begin{abstract}
Mitragynine (MG) and its major metabolites 7-hydroxymitragynine (7-OH-MG) are two of the major components of the plant extract Kratom, which is a tree planted in Southeast Asia. Kratom has long been used by opioid-dependent individuals as an alternative to their unavailable opioid of choice and chronic pain medication, as a stealth-to-urine drug screening opiate substitute while in opioid recovery treatment and recreationally, alone or as a booster. In this study, a direct infusion method was utilized and electrospray ionization triple quadrupole mass spectrometer was used as the detector for data acquisition. Pharmacokinetic study was conducted to investigate the effect of mitragynine and 7-hydroxymitragynine and major fragments of both compounds were proposed.
\end{abstract}

\section{Keywords}

Mitragynine, 7-Hydroxymitragynine, Kratom, HPLC-MS/MS, Pharmacokinetic, Mass Fragment

\section{Introduction}

Mitragynine (MG) and its major metabolites 7-hydroxymitragynine (7-OH-MG) are two of the major components of the plant extract Kratom [1] [2]. Kratom is derived from the Mitragyna speciose Korth tree in Southeast Asia, and like oxycodone, it has rare paradoxical properties consisting of augmented focus, concentration and physical energy, with concurrent reduction in physical and emotional pain [3] [4]. In the 1950s, mitragynine and its metabolites were prohibited by law in Thailand and in 2003 in Malaysia [5]-[8]. The U.S. Drug Enforcement 
Administration (USDEA) Office of Diversion Control lists kratom as a drug of concern; however, kratom remains legal in the U.S. and is also one of the most popular legal highs in the U.K. [9] [10]. M. speciose Korth contains more than 25 alkaloids that vary quantitatively depending on geographic location.

Several unique alkaloids are present in kratom leaves and the predominant alkaloid is mitragynine. Mitragynine (Figure 1) is structurally similar to the aphrodisiac yohimbine, which is the most prevalent of these alkaloids, and mitragynine is believed to be responsible for kratom's opioid effects [11] [12]. Another less prevalent alkaloid of kratom, 7-hydroxymitragynine, has also been studied for different functionality such as analgesic activity. 7-hydroxymitragynine is orally active in animals as an analgesic, and produces normal opioid side effects including constipation, with similar chemical structure to mitragynine compound.

In recent years, the hyphenated technique of chromatography and electrophoresis coupling with mass spectrometry has been widely used for different applications, for instance, kinetic study of cylindrospermopsin under $\mathrm{TiO}_{2}$ photocatalytic reaction [13], treatment of 6-hydroxymethyl uracil [14], Microcystis aeruginosa and microcystincyanotoxin [15], chiral separation of cathinone analogs [16] [17], microcystins separation and detection [18], and fluorescent substrate for monitoring acid phosphatase activity [19] [20].

In the previous study, it was focused on the method development and validation using LC-MS/MS [21]. A rapid and effective method for quantification of mitragynine and 7-hydroxymitragynine compounds in human urine matrix was reported. Correlation coefficients greater than 0.99 were obtained for both mitragynine and 7hydroxymitragynine compounds in the previous study [21]. In the current study, mass spectrometry part was emphasized with mass spectrum and mass fragments information. Pharmacokinetic and metabolites were examined using direct infusion electrospray ionization technique.

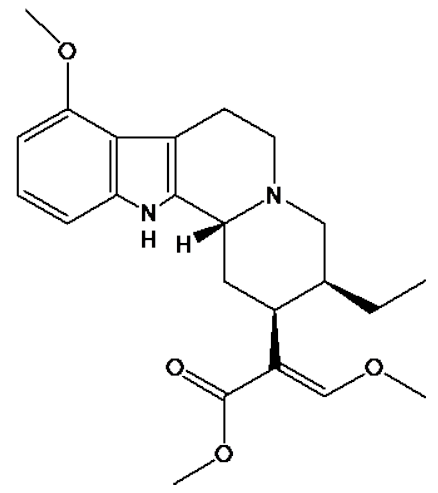

Mitragynine $\mathrm{C}_{23} \mathrm{H}_{30} \mathrm{~N}_{2} \mathrm{O}_{4}$ (m.w. 398.50)<smiles>CC[C@H]1CN2CCc3c([nH]c4cccc(OC(=O)OC)c34)[C@H]2C[C@H]1/C(=C\OC)C(=O)OC</smiles>

Mitragynine- $\mathrm{D}_{3}$ C23H27D3N2O4 (m.w. 401.51)<smiles>CC[C@H]1CN2CC[C@]3(O)C(=Nc4cccc(OC)c43)[C@@H]2C[C@@H]1/C(=C\OC)C(=O)OC</smiles>

7-Hydroxymitragynine $\mathrm{C}_{23} \mathrm{H}_{30} \mathrm{~N}_{2} \mathrm{O}_{5}$ (m.w. 414.49)<smiles>CC[C@H]1CN2CC[C@]3(O)C(=Nc4cccc(OC(=O)OC)c43)[C@@H]2C[C@H]1/C(=C\OC)C(=O)OC</smiles>

7-Hydroxymitragynine-D3 C23H27D3N2O5 (m.w. 417.51)

Figure 1. Chemical structures of mitragynine, mitragynine- $\mathrm{D}_{3}$, 7-hydroxymitragynine, and 7-hydroxy mitragynine- $\mathrm{D}_{3}$. 


\section{Experimental}

\subsection{Reagents}

Acetonitrile and HPLC-grade water are purchased from EMD Millipore (Billerica, MA, USA). Formic acid was purchased from Amresco (Solon, OH, USA). Mitragynine with a concentration of $100 \mu \mathrm{g} / \mathrm{mL}$ and 7-hydroxymitragynine with a concentration of $100 \mu \mathrm{g} / \mathrm{mL}$ standards were purchased from Cerilliant (Round Rock, TX, USA). Internal standards mitragynine- $\mathrm{D}_{3}$ with a concentration of $100 \mu \mathrm{g} / \mathrm{mL}$ and 7-hydroxymitragynine- $\mathrm{D}_{3}$ with a concentration of $100 \mu \mathrm{g} / \mathrm{mL}$ were purchased from Cerilliant (Round Rock, TX, USA). Internal standards were used for quantification purposes as a mean for correct the loss of analytes of interest during sample preparation process or sample injection. The phenyl-hexyl HPLC column was purchased from Phenomenex (Torrance, CA, USA).

\subsection{LC-MS/MS Instrumentation}

The assay was developed on a Shimadzu 20AD liquid chromatography (Columbia, MD, USA) coupled to an AB SciexQTrap 5500 quadrupole linear ion trap mass spectrometer (Framingham, MA, USA). A 2.6- $\mu \mathrm{m} 100 \mathrm{~mm} \times$ $2.1 \mathrm{~mm}$ phenyl-hexyl analytical column was employed, and gradient elution with a $0.4-\mathrm{mL} / \mathrm{min}$ flow rate of water and acetonitrile as mobile phases was utilized. The LC-MS/MS conditions were optimized to achieve rapid and effective goals for the detection of kratom compounds.

\subsection{Preparation of Standard Solutions}

Stock solutions were prepared weekly to keep the active component fresh. Methanol standards and urine standards were prepared for injection, respectively. The methanol standards were stored at $-8^{\circ} \mathrm{C}$, and the urine standards were stored at $4^{\circ} \mathrm{C}$. For HPLC injection, $50 \mu \mathrm{L}$ of working standards, $50 \mu \mathrm{L}$ of $10 \mathrm{ng} / \mathrm{mL}$ internal standards and $150 \mu \mathrm{L}$ of Mobile Phase A solution were mixed as the injection standards. For testing on urine samples, working standards were substituted with human urine.

\subsection{Sample Extraction}

Both blank and patient urine samples were stored at $-20^{\circ} \mathrm{C}$ until analysis. Urine samples were thawed and 1.0 $\mathrm{mL}$ aliquot was transferred to a $4 \mathrm{~mL}$ clear glass screw-top culture tube and spiked with $50 \mu \mathrm{L}$ of $10 \mathrm{ng} / \mathrm{mL}$ internal standards. The urine samples were centrifuged at 15,000 rpm speed for $15 \mathrm{~min}$ and the supernatant was transferred to a clue tube. With addition of internal standards and diluent, the mixture was transferred to an HPLC vial for HPLC-MS/MS analysis.

\subsection{Calibration}

Quantitation of mitragynine and 7-hydroxymitragynine were calibrated by internal standard technique. Deuterated internal standards purchased from Cerilliant (Round Rock, TX, USA) were added to the sample mixture as a calibration technique. The calibration curve was constructed by plotting the ratios of the peak area of mitragynine and mitragynine internal standard against the ratios of concentration of mitragynine and mitragynine internal standard. The $1 / x$ regression model was employed to acquire the regression equation and coefficient (r).

\section{Results and Discussion}

\subsection{HPLC Method Development}

Analytes were eluted with gradient mobile phases of water with $0.1 \%$ formic acid (Mobile Phase A) and acetonitrile (Mobile Phase B). Formic acid is a commonly-used additive for reversed-phase liquid chromatography, as it provides protons and promotes ionization for analytes. Acetonitrile is an organic solvent that provides advantages over methanol in terms of low back pressure, high sensitivity and less ghost peak for the gradient elution program.

The separation and identification of mitragynine and 7-hydroxymitragynine are demonstrated as Figure 2. The extracted chromatograms of both compounds are presented. As shown in the figure, 7-hydroxymitragynine eluted faster than mitragynine with the above mentioned conditions. 


\subsection{MS/MS Optimization}

The operating conditions and parameters for the electrospray ionization source were optimized to obtain the best mass spectrometric performance for both mitragynine and 7-hydroxymitragynine. The mass spectrometry parameters are listed as Table 1.

\subsection{Mass Spectra}

With the assistance of direct infusion electrospray ionization mass spectrometry technique, mass spectra and information such as molecular weight and mass fragment were obtained. Possible cleavage and metabolites mechanisms are proposed as Figure 3 for mitragynine and Figure 4 for 7-hydroxymitragynine. As indicated by the mass spectrum parameters in method development, the transition 174.3 is the major fragment of protonated mitragynine compound under instrument conditions stated in Table 1 and Table 2.

As illustrated in Figure 3, protonated mitragynine with $\mathrm{m} / \mathrm{z} 399.5$ has fragment of $\mathrm{m} / \mathrm{z}$ 174.3, 226.1 and 238.2. Figure 4 demonstrated the major fragment of protonated 7-hydroxymitragynine $\mathrm{m} / \mathrm{z} 415.5$ is $\mathrm{m} / \mathrm{z} 190.2$.

\subsection{Method Validation}

The analytical figures of merit were assessed and presented in Table 3. Linearity in terms of slope and Rsquared value was determined. Sensitivity including LOD and LOQ, intraday precision and interday precision were calculated as well. Limit of detection down to $0.0123 \mathrm{ng} / \mathrm{mL}$ for mitragynine and $0.0691 \mathrm{ng} / \mathrm{mL}$ for 7-hydroxymitragynine were achieved. The LOD values show that the established method is very sensitive for trace amount analysis.

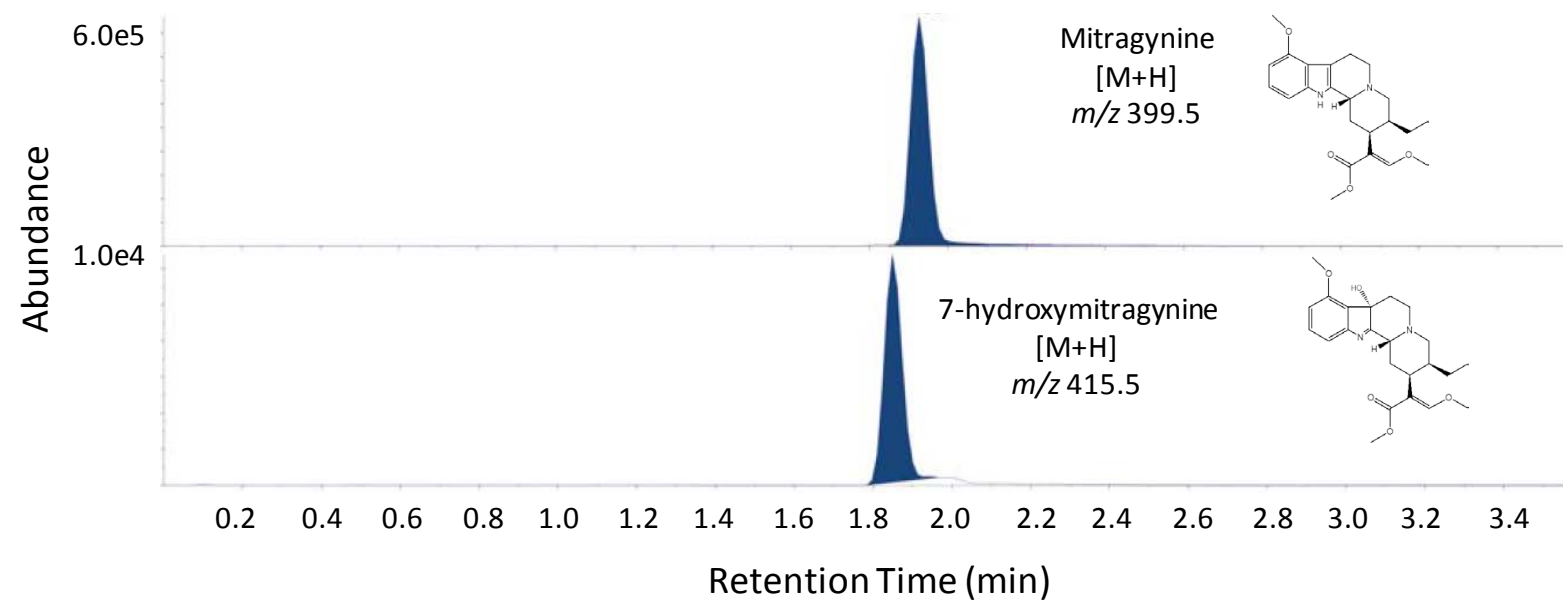

Figure 2. Extracted chromatograms of mitragynine and 7 -hydroxymotragyninewith $0.1 \%$ formic acid and acetonitrile as mobile phases.

Table 1. Optimized MS/MS operating conditions for mitragynine and 7-hydroxymigragynine obtained from tandem mass spectrometry.

\begin{tabular}{ccc}
\hline MS/MS conditions & Mitragynine & 7-hydroxymitragynine \\
Polarity & Positive & Positive \\
Ionspray voltage & $4000 \mathrm{~V}$ & $5000 \mathrm{~V}$ \\
Temperature & $550^{\circ} \mathrm{C}$ & $550^{\circ} \mathrm{C}$ \\
Collision gas & Medium & 5 edium \\
Ion source gas 1 & 50.0 & 55.0 \\
Ion source gas 2 & 65.0 & 65.0
\end{tabular}



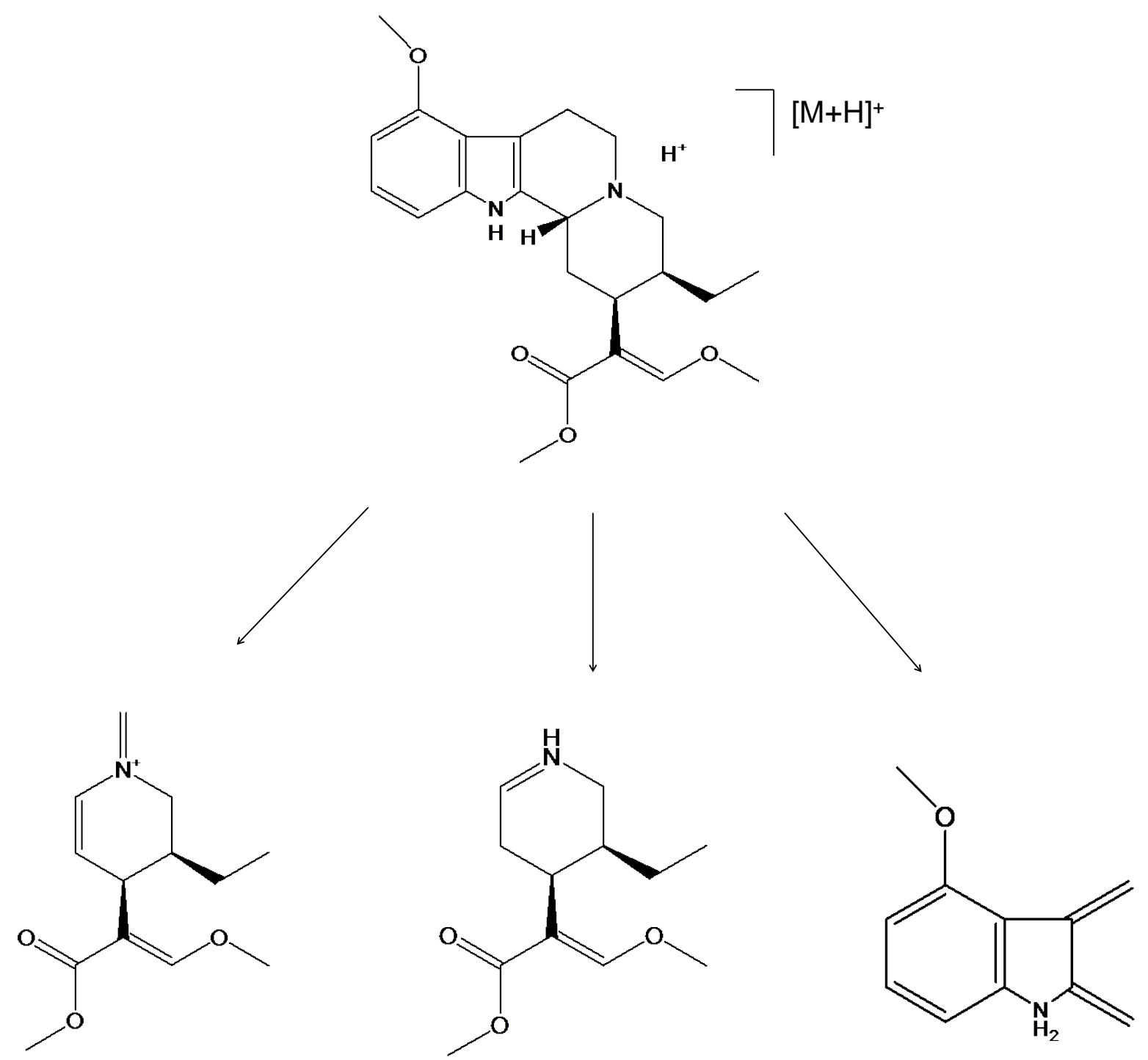

Figure 3. Mass fragmentation patterns of mitragynine.

Table 2. Multiple reaction monitoring (MRM) parameters of mitragynine and 7-hydroxymitragynine (analytes) and mitragynine- $\mathrm{D}_{3}$ and 7-hydroxymitragynine- $\mathrm{D}_{3}$ (internal standards).

\begin{tabular}{ccccc}
\hline MS/MS conditions & MG & 7-OH-MG & MG- $\mathrm{D}_{3}$ & 7-OH-MG- $\mathrm{D}_{3}$ \\
\hline Precursor ion $(\mathrm{m} / \mathrm{z})$ & 399.5 & 415.5 & 402.5 & 418.6 \\
Product ion $(\mathrm{m} / \mathrm{z})$ & 174.3 & 190.2 & 238.3 & 193.2 \\
Collision energy $(\mathrm{eV})$ & 45 & 45 & 35 & 45 \\
\hline
\end{tabular}

Table 3. Analytical figures of merit of LC-MS/MS results.

\begin{tabular}{|c|c|c|c|c|c|c|}
\hline \multirow{2}{*}{ Analytes } & \multicolumn{2}{|c|}{ Linearity } & \multicolumn{2}{|c|}{ Sensitivity } & \multirow{2}{*}{$\begin{array}{c}\text { Precision } \\
\text { (intraday, } \mathrm{n}=5 \text { ) } \\
\text { RSD } \%\end{array}$} & \multirow{2}{*}{$\begin{array}{c}\begin{array}{c}\text { Precision } \\
\text { (inter-day, } 3 \mathrm{~d} / \mathrm{n}=6 \text { ) }\end{array} \\
\text { RSD\% }\end{array}$} \\
\hline & Slope & $\mathrm{R}^{2}$ & LOD (ng/mL) & LOQ (ng/mL) & & \\
\hline Mitragynine & 92.5 & 0.9987 & 0.0123 & 0.0356 & 1.55 & 1.70 \\
\hline 7-hydroxymitragynine & 87.5 & 0.9951 & 0.0691 & 0.215 & 2.12 & 2.71 \\
\hline
\end{tabular}




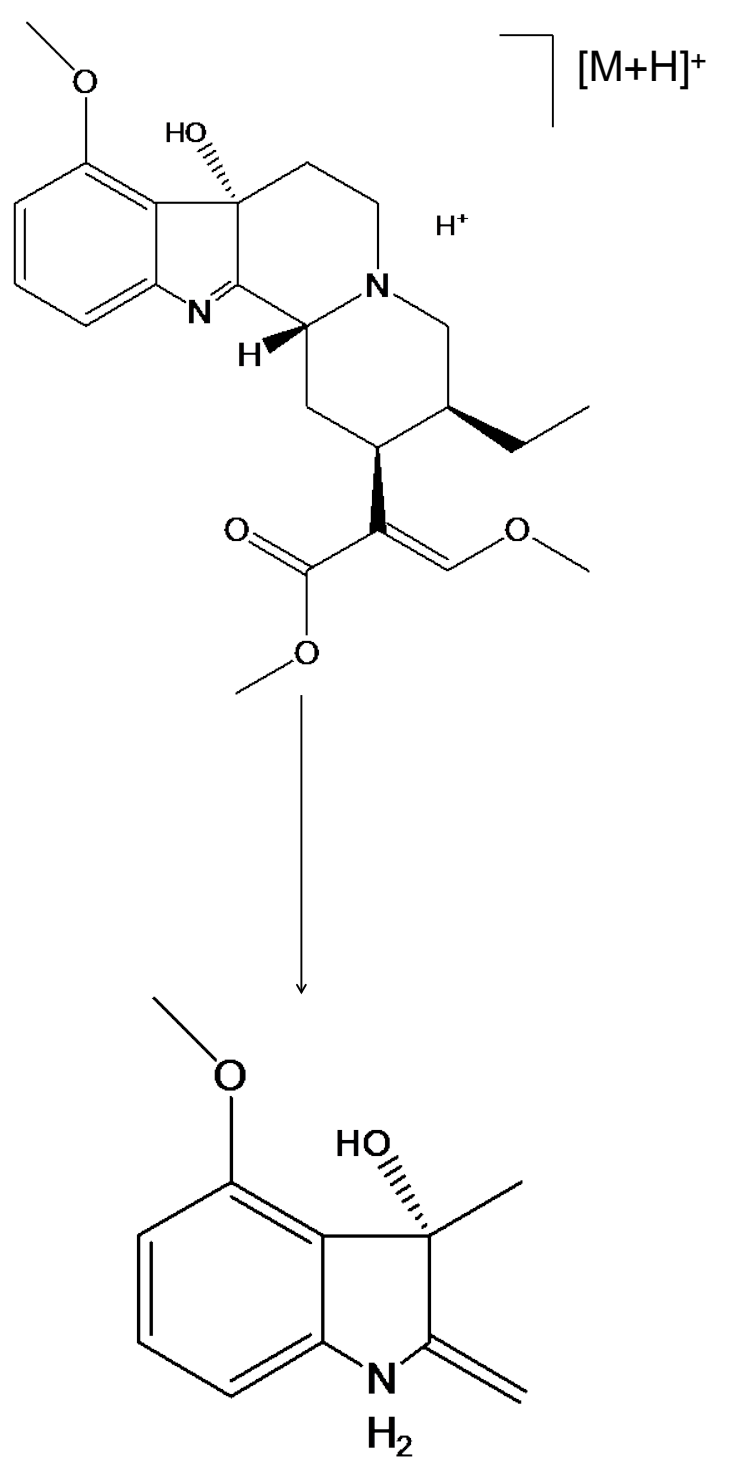

Figure 4. fragmentation patterns of 7-hydroxymitragynine.

\section{Concluding Remarks}

In this study, a specific detection and identification method by direct infusion electrospray ionization mass spectrometry has been developed for analysis of mitragynine and 7-hydroxymitragynine. The method demonstrates a rapid and precise route for the detection and identification in pharmaceutical and biomedical applications. This approach provides capability of mass spectrum and mass fragments information, which facilitates the pharmacokinetic study of mitragynine and 7-hydroxymitragynine compounds.

\section{References}

[1] Ward, J., Rosenbaum, C., Hernon, C., McCurdy, C. and Boyer, E. (2011) Herbal Medicines for the Management of Opioid Addiction. CNS Drugs, 25, 999-1007. http://dx.doi.org/10.2165/11596830-000000000-00000

[2] Suwanlert, S. (1975) A Study of Kratom Eaters in Thailand. Bulletin on Narcotics.

[3] Jansen, K.L.R. and Prast, C.J. (1988) Psychoactive Properties of Mitragynine (Kratom). Journal of Psychoactive Drugs, 20, 455-457. http://dx.doi.org/10.1080/02791072.1988.10472519

[4] Matsumoto, K., Mizowaki, M., Suchitra, T., Takayama, H., Sakai, S.-I., Aimi, N. and Watanabe, H. (1996) Antinoci- 
ceptive Action of Mitragynine in Mice: Evidence for the Involvement of Supraspinal Opioid Receptors. Life Sciences, 59, 1149-1155. http://dx.doi.org/10.1016/0024-3205(96)00432-8

[5] Vicknasingam, B., Narayanan, S., Beng, G.T. and Mansor, S.M. (2010) The Informal Use of Ketum (Mitragyna speciosa) for Opioid Withdrawal in the Northern States of Peninsular Malaysia and Implications for Drug Substitution Therapy. International Journal of Drug Policy, 21, 283-288. http://dx.doi.org/10.1016/j.drugpo.2009.12.003

[6] Boyer, E.W., Babu, K.M., Macalino, G.E. and Compton, W. (2007) Self-Treatment of Opioid Withdrawal with a Dietary Supplement, Kratom. The American Journal on Addictions, 16, 352-356. http://dx.doi.org/10.1080/10550490701525368

[7] Phongprueksapattana, S., Putalun, W., Keawpradub, N. and Wungsintaweekul, J. (2008) Mitragyna speciosa: Hairy Root Culture for Triterpenoid Production and High Yield of Mitragynine by Regenerated Plants. Zeitschrift für Naturforschung C: A Journal of Biosciences, 63, 691. http://dx.doi.org/10.1515/znc-2008-9-1014

[8] Zhao, C., Arroyo-Mora, L.E., DeCaprio, A.P., Sharma, V.K., Dionysiou, D.D. and O’Shea, K.E. (2014) Reductive and Oxidative Degradation of Iopamidol, Iodinated X-Ray Contrast Media, by Fe(III)-Oxalate under UV and Visible Light Treatment. Water Research, 67, 144-153. http://dx.doi.org/10.1016/j.watres.2014.09.009

[9] Babu, K.M., McCurdy, C.R. and Boyer, E.W. (2008) Opioid Receptors and Legal Highs: Salvia divinorum and Kratom. Clinical Toxicology, 46, 146-152. http://dx.doi.org/10.1080/15563650701241795

[10] Philipp, A.A., Wissenbach, D.K., Zoerntlein, S.W., Klein, O.N., Kanogsunthornrat, J. and Maurer, H.H. (2009) Studies on the Metabolism of Mitragynine, the Main Alkaloid of the Herbal Drug Kratom, in Rat and Human Urine Using Liquid Chromatography-Linear Ion Trap Mass Spectrometry. Journal of Mass Spectrometry, 44, 1249-1261. http://dx.doi.org/10.1002/jms.1607

[11] Kong, W.M., Chik, Z., Ramachandra, M., Subramaniam, U., Aziddin, R.E.R. and Mohamed, Z. (2011) Evaluation of the Effects of Mitragyna speciosa Alkaloid Extract on Cytochrome P450 Enzymes Using a High Throughput Assay. Molecules, 16, 7344-7356. http://dx.doi.org/10.3390/molecules16097344

[12] Chittrakarn, S., Keawpradub, N., Sawangjaroen, K., Kansenalak, S. and Janchawee, B. (2010) The Neuromuscular Blockade Produced by Pure Alkaloid, Mitragynine and Methanol Extract of Kratom Leaves (Mitragyna speciosa Korth.). Journal of Ethnopharmacology, 129, 344-349. http://dx.doi.org/10.1016/j.jep.2010.03.035

[13] Chen, L., Zhao, C., Dionysiou, D.D. and O’Shea, K.E. (2015) $\mathrm{TiO}_{2}$ Photocatalytic Degradation and Detoxification of Cylindrospermopsin. Journal of Photochemistry and Photobiology A: Chemistry, 307-308, 115-122. http://dx.doi.org/10.1016/j.jphotochem.2015.03.013

[14] Zhao, C., Pelaez, M., Dionysiou, D.D., Pillai, S.C., Byrne, J.A. and O’Shea, K.E. (2014) UV and Visible Light Activated $\mathrm{TiO}_{2}$ Photocatalysis of 6-Hydroxymethyl Uracil, a Model Compound for the Potent Cyanotoxin Cylindrospermopsin. Catalysis Today, 224, 70-76. http://dx.doi.org/10.1016/j.cattod.2013.09.042

[15] Liu, S., Zhao, Y., Ma, F., Ma, L., O’Shea, K., Zhao, C., Hu, X. and Wu, M. (2015) Control of Microcystis aeruginosa Growth and Associated Microcystin Cyanotoxin Remediation by Electron Beam Irradiation (EBI). RSC Advances, 5, 31292-31297. http://dx.doi.org/10.1039/C5RA00430F

[16] Merola, G., Fu, H., Tagliaro, F., Macchia, T. and McCord, B.R. (2014) Chiral Separation of 12 Cathinone Analogs by Cyclodextrin-Assisted Capillary Electrophoresis with UV and Mass Spectrometry Detection. Electrophoresis, 35, 3231-3241. http://dx.doi.org/10.1002/elps.201400077

[17] Merola, G., Fu, H., Tagliaro, F., Macchia, T. and McCord, B. (2014) Chiral Separation of 12 Cathinone Analogs by Cyclodextrin-Assisted Capillary Electrophoresis Time-of-Flight Mass Spectrometry. 41st International Symposium and Exhibit on High Performance Liquid Phase Separations and Related Techniques (HPLC 2014), New Orleans.

[18] Zheng, B., Fu, H., Berry, J.P. and McCord, B. (2016) A Rapid Method for Separation and Identification of Microcystins Using Capillary Electrophoresis and Time-of-Flight Mass Spectrometry. Journal of Chromatography A, 1431, 205-214. http://dx.doi.org/10.1016/j.chroma.2015.11.034

[19] Yang, D., Li, Z., Diwu, Y.A., Fu, H., Liao, J., Wei, C. and Diwu, Z. (2008) A Novel Fluorogenic Coumarin Substrate for Monitoring Acid Phosphatase Activity at Low pH Environment. Current Chemical Genomics, 2, 48. http://dx.doi.org/10.2174/1875397300802010048

[20] Fan, M., Yang, D., Wang, X., Liu, W. and Fu, H. (2014) DOSS-Based QAILs: As Both Neat Lubricants and Lubricant Additives with Excellent Tribological Properties and Good Detergency. Industrial \& Engineering Chemistry Research, 53, 17952-17960. http://dx.doi.org/10.1021/ie502849w

[21] Fu, H., Cid, F.X., Dworkin, N., Cocores, J. and Shore, G. (2015) Screening and Identification of Mitragynine and 7Hydroxymitragynine in Human Urine by LC-MS/MS. Chromatography, 2, 253-264. http://dx.doi.org/10.3390/chromatography2020253 


\section{Submit or recommend next manuscript to SCIRP and we will provide best service for you:}

Accepting pre-submission inquiries through Email, Facebook, Linkedin, Twitter, etc A wide selection of journals (inclusive of 9 subjects, more than 200 journals)

Providing a 24-hour high-quality service

User-friendly online submission system

Fair and swift peer-review system

Efficient typesetting and proofreading procedure

Display of the result of downloads and visits, as well as the number of cited articles

Maximum dissemination of your research work

Submit your manuscript at: http://papersubmission.scirp.org/ 